\title{
REGISTRO DE POMACEA CANALICULATA (LAMARCK, 1822) (AMPULLARIIDAE), MOLUSCO EXOTICO PARA EL NORTE DE CHILE
}

\author{
RECORD OF POMACEA CANALICULATA (LAMARCK, 1822) \\ (AMPULLARIIDAE), EXOTIC MOLLUSK FOR TO NORTH CHILE
}

\author{
Douglas Jackson ${ }^{1} \&$ Donald Jackson ${ }^{2}$ \\ ${ }^{1}$ Sociedad Chilena de Entomología, Casilla 21132, Santiago (21), Chile. sillitus@ hotmail.com, \\ ${ }^{2}$ Departamento de Antropología, Facultad de Ciencias Sociales, Universidad de Chile. Casilla 10115, Santiago, Chile. \\ djackson@uchile.cl.
}

\begin{abstract}
In this paper we inform about the presence in the coast of Choapa (IV Region of Coquimbo, Chile), of a non-local freshwater mollusk -Pomacea canaliculata (Lamarck, 1822) (Ampullariidae). We describe its morphology, habitat and relations with other freshwater mollusks. We also discuss its potential role as link to the zoonosis parasitaría.
\end{abstract}

Keywords: Pomacea canaliculata (Lammarck), Norte de Chile, Nuevo registro

\section{RESUMEN}

En este trabajo informamos acerca de la presencia en la costa de Choapa (IV Región de Coquimbo, Chile), de un molusco de agua dulce Pomacea canalículata (Lammarck, 1822) (Ampullariidae). Se describe la morfología, habitat y relaciones con otros moluscos de agua dulce. Se discute su potencial rol con relación a la zoonosis parasitaria.

Palabras Clave: Pomacea canaliculata (Lammarck), Norte of Chile, new record.

\section{INTRODUCCION}

La familia Ampullariidae (Prosobranchia), agrupa moluscos dulceacuícolas que se encuentran en Africa, Asia y América (Martin \& Negrete 2007), muy utilizados en acuarios comerciales (Pereda \& Walls 1996). En América se encuentran 43 géneros (Cowie \& Thiengo 2003), ninguno de ellos representados en Chile.

El genero Pomacea Perry 1810, se distribuye principalmente en la Region Neotropical, siendo este género frecuente en lagunas y ríos de Sudamérica, especialmente en Argentina (Gamarra-Luques et al. 2004, Rumi et al. 2008) y Uruguay (Scarabino 2004). Por otra parte, algunos registros paleontológicos muestran la existencia de Pomacea canaliculata en la Formación Dolores y Sopas de Uruguay datada entre los 43,000 y 45,000 años A.P. (Martínez \& Rojas 2004), así como también ejemplares de Pomacea sp., datados directamente en 3.350+-50 años A.P. del período Platense de la Provincia de Buenos Aires en Argentina (Toledo 2005).

La importancia de la detección de este molusco herbívoro, radica en su eventual impacto sobre las comunidades de plantas acuáticas, como también por su importancia medica al ser un hospedero intermediario del nematodo, Angiostrongylus cantotensis (Thiengo 1995); aunque también existen especies comestibles que tienen gran importancia comercial, como Pomacea patula catemacensis 
(Baker 1922), que se encuentra en México (Carreón et al. 2003).

En Chile es el único representante de la familia y el primero en detectarse en ambientes silvestres, no obstante que Pomacea bridgessi (Reeve 1856) se había registrado en acuarios comerciales (Letelier et al. 2007).

El objetivo de este reporte es dar a conocer el registro del "Caracol Manzana", Pomacea canaliculata (Lamarck 1822), en la cuenca hidrográfica de la provincia del Choapa, especie de Ampullariidae no representada hasta ahora en ambientes acuáticos de Chile (Valdovinos 2006) y describir el habitat de este nuevo registro en América.

\section{MATERIAL Y METODO}

El área del hallazgo se encuentre en el curso inferior del estero Conchalí, situado aproximadamente a 4,5 $\mathrm{Km}$. al norte de Los Vilos ( $31^{\circ} 55^{\prime} \mathrm{LW}, 71^{\circ} 31^{\prime} \mathrm{LS}$ ), en el límite sur de la provincia de Choapa, en la IV Región de Coquimbo. Este estero constituye una pequeña cuenca hidrográfica cuyo nacimiento se encuentra a unos $50 \mathrm{Km}$. al interior de la localidad de Mauro (Comuna de Los Vilos).

Las muestras se encontraron en febrero (2009), en el curso inferior del estero de Conchalí y en la desembocadura de la laguna denominada con el mismo nombre, que se encuentra aproximadamente a $1 \mathrm{Km}$. de la línea litoral.

Tanto los especimenes vivos como muertos $(\mathrm{N}=38)$ de Pomacea canaliculata, se recuperaron manualmente desde la riberas del estero y laguna, donde se encontraban entre la vegetación acuática o desplazándose sobre el fondo lodoso. También se recolectaron algunas masas ovígeras que se encontraban adheridas a la vegetación ribereña que fueron fijadas junto a 25 ejemplares adultos en alcohol al $75 \%$.

Adicionalmente se tomaron muestras $(\mathrm{N}=64)$ de la vegetación acuática y de los sedimentos, los cuales fueron tratados por el método de flotación con malla de $2 \mathrm{~mm}$ de abertura, para recuperar estados inmaduros de $P$. canaliculata y eventuales micromoluscos asociados a esta especie. También se disectaron tres ejemplares de Pomacea canaliculata para la búsqueda de posibles parásitos, lo cual se realizo bajo una lupa estereoscópica Nikon, con oculares de 10x/21 y objetivo zoom de $0,66 \times 4$.

Las mediciones fueron realizadas con un pie de metro (precisión de $0.05 \mathrm{~mm}$ ) sobre ejemplares adultos, para lo cual se considero la longitud total y ancho máximo de la concha, como también su abertura. Similares medidas se registraron en los opérculos, masas ovígeras y huevos.

La temperatura del agua se registró con un termómetro manual y para determinar de $\mathrm{su} \mathrm{pH}$, con papel indicador universal de escala 1-14 (Macherey-Nagel).

Para la identificación se extrajo la rádula y se comparo con cinco especies diferentes del género Pomacea (Martín y Negrete, 2007). Para la descripción de la concha se trabajo con las descripciones de Pereda \& Walls (1996) y Thiengo et al. (1993).

El estudio morfológico de ejemplares en diferentes estados de desarrollo incluyo el examen de la estructura de la rádula, permitiendo establecer claramente que la especie presente en Chile es Pomacea canaliculata (Lamarck 1822).

Los especimenes estudiados quedan depositados en la Sección de malacología del Museo Nacional de Historia Natural de Santiago y en la colección del Departamento de Antropología de la Facultad de Ciencias Sociales de la Universidad de Chile.

\section{RESULTADOS}

Pomacea canaliculata, se caracteriza morfológicamente por presentar una concha globosa muy grande (Fig.1 A) que puede alcanzar una longitud total de más de $70 \mathrm{~mm}$, el último anfracto dos veces más largo que la longitud de la espira (en vista dorsal). La abertura es dextra, grande, oval ligeramente alargada, con margen del labio externo simple. El ombligo es evidente en juveniles y adultos, de abertura amplia y profunda, que llega a nivel medio del labio parietal. La espira es baja de cinco vueltas, la sutura es profunda en forma de canal, carácter que fundamenta el nombre especifico de este taxón.

La concha es delgada, de coloración normalmente café oscuro que puede variar a beige, con textura cubierta de líneas de crecimiento axiales y radiales que forman un reticulado tenue, que puede estar enmascarada por una costra de lodo, pero siempre es evidente en la superficie ventral del último anfracto (fig. $1 \mathrm{~B}$ ). El labio columelar es levemente curvo, cubriendo parcialmente el ombligo; el labio parietal sin callo de unión. El opérculo (fig. 1 C) es grueso, 
translucido, con espira nuclear excéntrica y líneas de crecimiento con bandas intercaladas de color café oscuro y claro, superficie internamente brillante de color negro a café oscuro; su longitud varia entre 39,40 y 26,70 mm ( $\mathrm{N}=5$ ejemplares: Promedio $=$ 33,10; Desviación Estándar $=4,89)$ y su ancho máximo entre 26,80 y $17,40 \mathrm{~mm}(\mathrm{~N}=5$ ejemplares: Promedio = 22,80; Desviación Estándar = 3,51).

Rádula con hilera de dientes centrales largos, base más ancho que larga, cúspide casi trapezoidal, con tres conos laterales.

Tamaño en mm de los especimenes estudiados $(\mathrm{N}=$ 38): Longitud total de 19,12 a 73,74 (Promedio = 51,73; Desviación Estándar = 14,09). Ancho máximo de 16,06 a 63,01 (Promedio = 43,48; D. Estándar $=12,22$ ). Largo abertura de 14,50 a 50,70 $($ Promedio $=35,20 ;$ D. Estándar $=8,69)$. Ancho abertura de 10,80 a 46 (Promedio $=28,30 ; \mathrm{D}$. Estándar = 8,04).

Los huevos se agrupaban en masas ovígeras en forma de racimos de color rozado y tienen un diámetro promedio máximo de 33,84 mm (Promedio de $31,18 \mathrm{~mm}$ ). Cada masa ovígera contienen un promedio de 324 huevos $(\mathrm{N}=2)$; las que se encuentran adheridas a la superficie aérea de plantas acuáticas y en rocas que se hallaban en la ribera del estero y laguna de Conchalí (Fig. 2).
Los huevos son de color rozado que al contacto al aire se calcifican, al igual que el de otras especies del género (Pereda \& Walls 1996). El diámetro máximo de los huevos varia entre 3,02 a $3,35 \mathrm{~mm}(\mathrm{~N}=40$ : Promedio = 3,15; Desviación Estándar = 0,09)

El habitat donde se recolectaron las muestras de Pomacea canaliculata, esta compuesta por una vegetación ribereña donde se destacan los totorales (Typha angustifolia) y por plantas acuáticas con hojas natantes, como Jussiaea repens L. (Decodontidae). El sistema acuático corresponde a aguas lenticas, poco profundas $(60 \mathrm{~cm})$, con un $\mathrm{pH}$ neutro (7) y temperaturas que varian entre los 18 y $20^{\circ} \mathrm{C}$.

Dentro de los moluscos dulceacuícolas asociados a $P$. canaliculata, se encontraron los géneros Pisidium $(\mathrm{N}=25)$, Littoridina $(\mathrm{N}=22)$ y Physa $(\mathrm{N}=8)$.

Los 25 ejemplares colectados vivos durante la mañana del día 21 de febrero (2009), se hallaban muy activos sobre las plantas acuáticas y sobre el fondo lodoso, encontrándose algunos especimenes en copula, mientras que otros se alimentaban de Jussiaea repens L., cuyas hojas presentan los márgenes foliares consumidos.

La densidad observada es de aproximadamente de 8 a 10 ejemplares por $1 \mathrm{mt}$, no obstante de ser gregarios se disponen en forma dispersa.

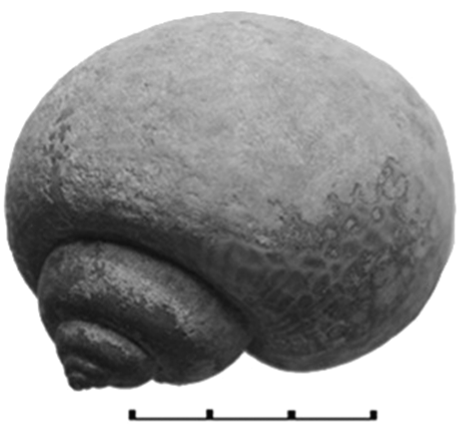

A

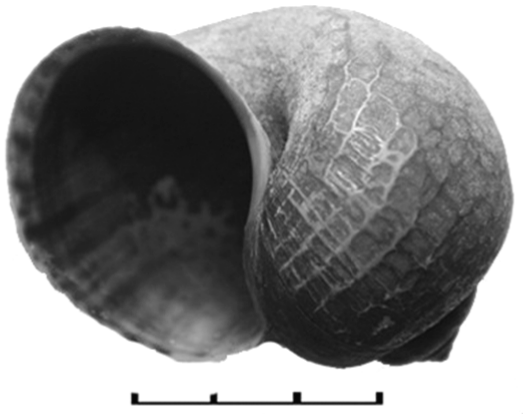

B

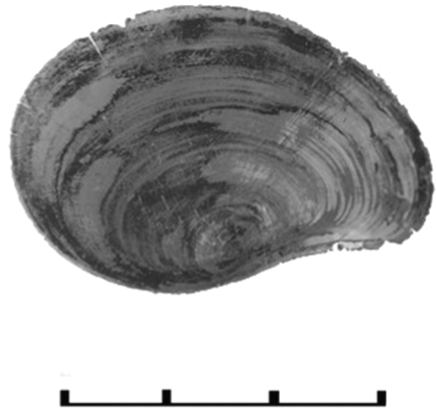

C

Figura. 1. Pomacea canaliculata (Lamarck, 1822), A: Vista dorsal, B: Vista oral y C: opérculo (Escala: $3 \mathrm{cms}$ )

Figure. 1. Pomacea canaliculata (Lamarck, 1822), A: oral view, B: view y C: operculum (Scale: $3 \mathrm{~mm}$ ) 
Registro de Pomacea canaliculata, molusco exótico para el norte de Chile: JACKsON D. \& JACKsON D.

Por otra parte, se recolectaron 13 ejemplares adultos muertos, de los cuales siete tienen huellas de fracturas o perforaciones en la concha, lo que sugiere su consumo por aves, probablemente por garzas (Bubulcus ibis, Egretta thula) o Huairavos (Nycticorax nycticorax) que frecuentan el estero y la laguna Conchalí.

El examen del intestino de tres ejemplares adultos de Pomacea canaliculata mostraron la presencia de fragmentos estructurales de plantas acuáticas (hojas y tallos) y otros restos orgánicos no identificables. Un singular hallazgo en los intestinos fue el encuentro de micro moluscos que incluyen Littoridina sp. (Hidrobiidae), Physa sp. (Physidae) y Pisium sp. (Sphaeriidae), moluscos que probablemente ingresaron al tracto digestivo en forma pasiva a través de los vegetales consumidos.

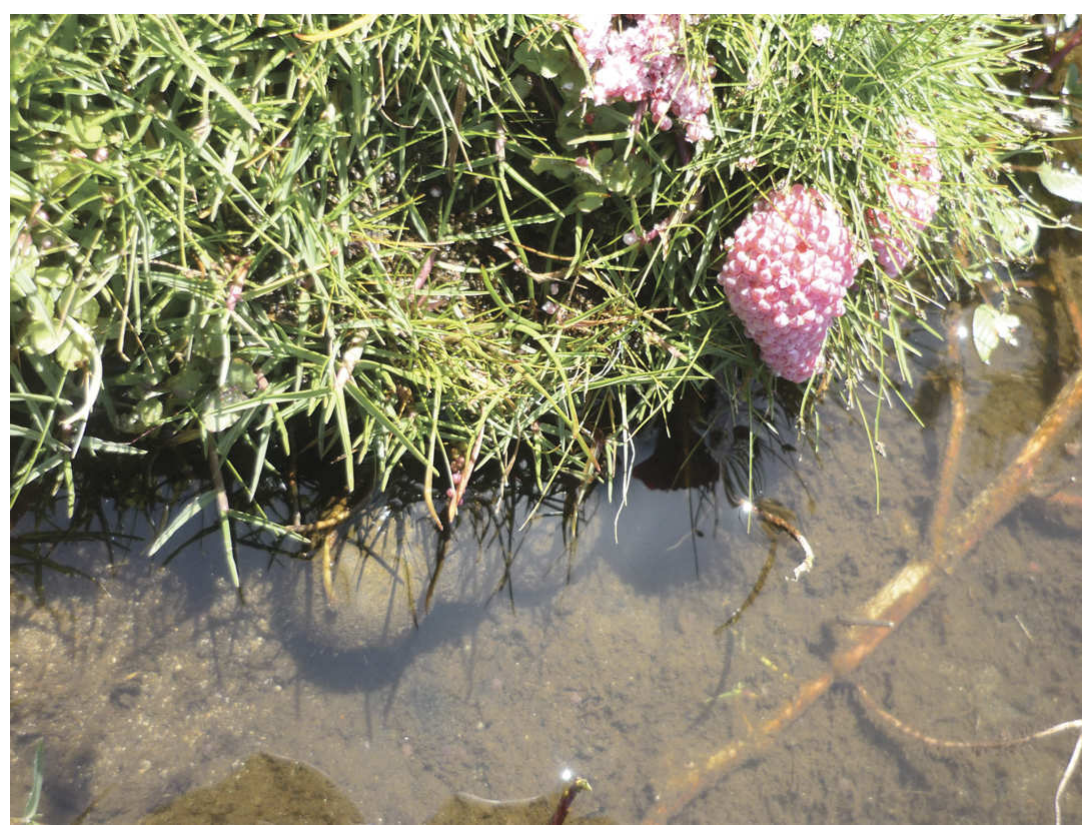

Figura. 2: Masas ovígeras in situ de Pomacea canaliculata (Lamarck, 1822) colectados en el Estero de Conchalí.

Figure. 2. Mass ovigeras in situ of Pomacea canaliculata (Lamarck, 1822) collected in the Estero Conchalí.

\section{DISCUSION Y CONCLUSIONES}

Es una especie inconfundible por su gran tamaño, espira con suturas profundas y presencia de opérculo, caracteres que en conjunto no son comparables a ninguna especie nativa del país, incluso en ejemplares inmaduros de $3 \mathrm{~mm}$ longitud, pueden ser identificables por las suturas en las espiras y un evidente ombligo. Este molusco es una especie dioica de fecundación interna y desarrollo ovíparo, como así lo demuestra el haber observado ejemplares en copula y masas ovígeras, sin embargo aún no podemos diferenciar sexos por la morfología de la concha, aunque internamente hemos observado la glándula de albúmina de coloración naranjo intenso, que diferencia claramente a las hembras maduras, de aspecto similar a lo observado en $P$. patula catemacensis (Carreón et al. 2003).

La inspección de varios sectores a lo largo de los ríos Choapa y río Illapel, como también en el curso 
superior del estero Conchalí y localidades como Pichidangui y Chigualoco, no se detectaron especimenes de Pomacea canaliculata, lo que confirma la reciente introducción de este molusco en este estero.

Probablemente la introducción de esta especie provenga del territorio Argentino a través del flujo de turistas hacia la Serena, lo que debió ocurrir posterior al año 2007, fecha en que no estaba presente. El recambio de agua en acuarios que transportan acuaristas podría ser el mecanismo de introducción, como así se ha registrado $P$. bridgesii (Reeve, 1856) en acuarios comerciales en nuestro país (Letelier et al. 2007).

Finalmente se debe alertar a las autoridades sanitarias, que en las muestras estudiadas de este molusco introducido, no se han detectado parásitos de importancia medica, aunque en Asia, Cuba y Malasia se ha detectado a Angiostrongylus cantonensis (Chen 1935), nematodo que utiliza como huéspedes intermediarios a $P$. canaliculata (Lamarck 1822) y P. paludosa (Say 1829), (Letelier et al. 2007). Por otra parte tampoco se detectó el copépodo Argentino, Ozmana huarpium (Ozmanidae), especie endosimbionte de P. canaliculata a (Gamarra-Luques et al. 2004)

\section{AGRADECIMIENTOS}

Eileen Riedemann por el trabajo fotográfico, así como a la Dra. Cecilia Osorio de la Facultad de Ciencias de Universidad de Chile, por la lectura del manuscrito inicial.

\section{BIBLIOGRAFIA}

Carreón L., E. Uria, F. Espinosa \& F. Martínez. 2003. Desarrollo morfológico del sistema reproductor de Pomacea patula catemacensis (Baker 1922) (Mollusca, Caenogastropoda: Ampullariidae).
Revista Chilena de Historia Natural 76: 665-680

Cowie R. \& Thiengo, Sc. 2003. The apple snails of the Ameritas (Mollusca: Gastropoda: Ampullariidae: Asolene, Felipponea, Marisa, Pomacea, Pomella): a nomeclatural and type catal. Malacología 45: 41-100.

Gamarra-luques C., I. Vega, E. Koch \& A.castroVAZQUEZ. 2004. Intrahost distribution and trasmisión of anew species of cyclopoid copepod endosymbiotic to a freshwater snail, Pomacea canaliculata (Caenogastropoda, Ampullariidae) from Argentina. Biocell 28 (3): 155-164.

Letelier S., A. Ramos \& L. Huaquín. 2007. Moluscos dulceacuícolas exóticos en Chile. Revista Mexicana de Biodiversidad 78:9-13.

Martín S. \& Negrete L. 2007. Radular ultrastructurre of South American Ampullariidae (Gastropoda: Prosobranchia). Brazilian Journal Biological., 67 (4):721-726.

Martínez S. \& A. RoJas. 2004. Quaternary continental molluscs from Northern Uruguay: distribución and paleoecology. Quaternary International 114:123-128.

Perera G. \& J. Walls. 1996. Apple Snails in the Aquarium. Ed. T. F. H. Publicaciones, Inc.

Rumi A., D. Gutiérrez, V. NúÑEz \& G. Darrigan. 2008. Malacología Latinoamericana. Moluscos de agua dulce de Argentina. Revista de Biología Tropical. (Int. J. Trop. Biol.), 56 (1): 77-111.

SCARABINo, F. 2004. Lista sistemática de los gastropoda dulceacuícolas vivientes de Uruguay. Comunicaciones de la Sociedad Malacológica del Uruguay 8 (84-85 /86-87) : 347-356.

Thiengo Sc., C. Borda, Araújo \& J.L. Barros. 1993. On Pomacea canaliculata (Lamarck, 1822) (Mollusca: Pilidae). Memôrias do Instituto Oswaldo Cruz 88 (1): 67-71

Thiengo Sc., 1995. Género Pomacea (Perry, 1810). In tópicos en Malacología Medica. Frederico Simoes Barbosa. Fiocruz, Rio de Janeiro pp. 53-69

Toledo, M. 2005. Secuencias pleistocenas "Lujanenses" ensu sección tipo: Primeras dataciones $14 \mathrm{C}$ e implicancias estratigráficas, arqueológicas e históricas, Luján-Jáuregui, Provincia de Buenos Aires. Revista de la Asociación Geológica Argentina 60(2):1-14.

VAlDovinos, C. 2006. Estado de conocimiento de los gastrópodos dulceacuícolas de Chile. Gayana 70 (1): 88-95. 